\title{
SUSCETIBILIDADE DE HELICOVERPA ZEA (BODDIE) (LEP.: NOCTUIDAE) A BACILLUS THURINGIENSIS BERLINER (BACILLACEAE)
}

\section{H.J.G. dos Santos Junior'1, E.J. Marques ${ }^{2}$, R.A. Polanczyk ${ }^{1}$, D. Pratissolii ${ }^{1}$, V.M. Rondelli ${ }^{2}$}

${ }^{1}$ Universidade Federal do Espírito Santo, Centro de Ciências Agrárias, Departamento de Produção Vegetal/ Núcleo de Desenvolvimento Científico e Tecnológico em Manejo Fitossanitário, Alto Universitário, s/nō, CEP 29500-000, Alegre, ES, Brasil. E-mail: hjsantosjr@hotmail.com

\section{RESUMO}

A utilização de agentes de controle biológico é uma alternativa para reduzir o impacto ocasionado pela adoção do uso intensivo de produtos químicos sintéticos, além de possibilitar a produção de alimentos mais saudáveis e que possam gerar mais renda ao produtor. A bactéria entomopatogênica Bacillus thuringiensis Berliner é uma excelente opção, uma vez que é específica e não é prejudicial ao homem. Este trabalho teve como objetivo avaliar 27 isolados de $B$. thuringiensis obtidos a partir de amostras de solos e duas formulações comerciais (Dipel ${ }^{\circledR}$ PM e Xentari ${ }^{\circledR}$ WG) visando sua utilização em programas de manejo de Helicoverpa zea (Boddie), analisando suscetibilidade, efeito subletal e a toxicidade por meio de estimativas da $\mathrm{CL}_{50}$ em lagartas de primeiro instar. Os isolados B.t11, B.t21, B.t 23, B.t25, B.t26, B.t 27, B.t 28 e os formulados proporcionaram mortalidades superiores a $94 \%$, e os demais isolados ocasionaram mortalidades inferiores a $33,33 \%$. A CL $\mathrm{L}_{50}$ variou entre $3 \times 10^{4}$ a $1,2 \times 10^{7}$ esporos $/ \mathrm{mL}$ de B. thuringiensis. Os isolados B.t 6, B.t12, B.t15, B.t 16 e B.t 18 afetaram o desenvolvimento deH. zea, reduzindo o peso de lagartas e pupas. Entre os isolados, B.t 23 pode ser promissor para o controle de lagartas de H. zea.

PALAVRAS-CHAVE: Controle microbiano, bactéria entomopatogênica, lagarta-da-espiga.

\section{ABSTRACT}

SUSCEPTIBILITY OF HELICOVERPA ZEA (BODDIE) (LEP.: NOCTUIDAE) TO BACILLUS THURINGIENSIS BERLINER(BACILLACEAE). The use of biological control agents is an alternative that can reduce the impact of intensive use of synthetic chemicals, therefore allowing for production of healthier food while generating more revenue for the growers. The entomopathogenic Bacillus thuringiensis Berliner $(\mathrm{Bt})$ is an excellent option due to its specificity and harmlessness to humans. The present study investigated the susceptibility, toxicity and sublethal effects of $27 \mathrm{~B}$. thuringiensis isolates obtained from soil samples and 2 commercial formulations (Dipe ${ }^{\circledR}$ PM and Xentari ${ }^{\circledR}$ WG) to first-instar larvae of Helicoverpa zea (Boddie) (Lep.: Noctuidae). The Bt isolates B.t 11, B.t 21, B.t 23, B.t 25, B.t 26, B.t 27, B.t 28 and the commercial Bt formulations caused larval mortalities of over $94 \%$. The other isolates caused mortalities under $33.33 \%$. The $\mathrm{LC}_{50}$ ranged from $3 \times 10^{4}$ to $1.2 \times 10^{7}$ spores $\mathrm{mL}^{1}$. In addition, the isolates B.t 6, B.t 12, B.t 15, B.t 16 and B.t 18 caused reduction in the weight of $H$. zea larvae and pupae. Among the isolates, B.t 23 has the potential to be used for the control of $H$. zea larvae.

KEY WORDS: Microbiological control, entomopathogen bacteria, corn earworm.

\section{INTRODUÇÃO}

Insetos-pragas, entre os quais Helicoverpa zea (Boddie) (Lepidoptera: Noctuidae), causam prejuízos em inúmeras culturas como, por exemplo, o algodão, milho e tomate que são consideradas culturas de importância sócio-econômica Gould et al., 2002; NAUlt;SPEESE III, 2002; Giolo et al., 2006; LeBEDENCO et al., 2007). No milho, infestações de $H$. zea podem chegar a $90 \%$ das espigas, reduzindo em até $8 \%$ a produtividade. No Brasil, alguns plantios de tomate sofrem danos da ordem de até $80 \%$, inviabilizando a produção, quando o controle com agrotóxicos não é adotado (França et al., 2000; PinTo et al., 2004).

O uso de inseticidas sintéticos é a principal tática para o controle de $H$. zea, porém a necessidade de produção de alimentos mais saudáveis vem estimulando e favorecendo o uso de medidas alternativas

\footnotetext{
${ }^{2}$ Universidade Federal Rural de Pernambuco, Departamento de Agronomia - Entomologia, Recife, PE, Brasil.
} 
aos inseticidas químicos. Entre essas, a utilização de agentes de controle biológico com a bactéria entomopatogênica Bacillus thuringiensis Berliner (Eubacteriales: Bacillaceae). (Glare; O'CALlaghan, 2000; KARIM etal., 2000;B OBROWSKI et al., 2003; POLANCZYK; Alves, 2003).

Entretanto, para obter sucesso na utilização de $B$. thuringiensis em programas de manejo de insetospraga, é necessária a adoção de isolados eficientes. Logo, a seleção de isolados nativos pode fortalecer o desenvolvimento regional, uma vez que a maioria dos produtos disponíveis no mercado é importada e onera os custos de produção (PoLANCZYK et al., 2008).

A respeito deste assunto, trabalho realizado por Rogoff et al. (1969) é um exemplo clássico, onde os autores avaliaram o potencial de diferentes isolados de $B$. thuringiensis visando o controle de alguns insetos-praga, dentre esses $H$. zea. A seleção de isolados de $B$. thuringiensis e, consequentemente, a descoberta de novas proteínas com atividade inseticida é uma forma de incrementar o manejo de insetos-praga (EsCUdERo et al., 2004; MEdeIRos et al., 2005).

Portanto, estudos que avaliem a suscetibilidade de insetos-praga a novos isolados de B. thuringiensis são de primordial relevância. Desta forma, este trabalho teve como objetivo selecionar o(s) isolado(s) de $B$. thuringiensis que apresentem potencial para o controle de $H$. zea, para serem incorporados em programas de manejo integrado.

\section{MATERIAL E MÉTODOS}

O trabalho foi conduzido no Núcleo de Desenvolvimento Científico e Tecnológico em Manejo Fitossanitário (NUDEMAFI) do Centro de Ciências Agrárias da Universidade Federal do Espírito Santo (CCA-UFES), Alegre, ES, em sala climatizada ajustada para a temperatura de $26 \pm 1^{\circ} \mathrm{C}, 70 \pm 10 \%$ de umidade relativa e fotofase de $12 \mathrm{~h}$.

Coleta e criação de $H$. zea. A coleta de $H$. zea foi realizada em plantios de milho, no Município de Alegre, ES (20 $\left.45^{\prime} 50^{\prime \prime} S 41^{\circ} 31^{\prime} 58^{\prime \prime} \mathrm{O}\right)$, e a criação foi desenvolvida em sala climatizada $\left(26 \pm 2^{\circ} \mathrm{C}\right.$, UR de 60 $\pm 10 \%$ e fotofase de $12 \mathrm{~h}$ ). Os adultos de $H$. zea foram mantidos em gaiolas de PVC ( $20 \mathrm{~cm}$ de diâmetro $x 25$ $\mathrm{cm}$ de altura) revestidas internamente com folha de papel branco, com as extremidades fechadas com tecido do tipo "voil", sendo oferecida diariamente uma solução de mel a 10\% como substrato alimentar. Os ovos de $H$. zea foram coletados e acondicionados em recipientes plásticos e, após a eclosão, as lagartas foram individualizadas em tubos de vidro $(8,5 \times 2,5$ $\mathrm{cm}$ ) preenchidos até em $1 / 4$ de seu volume com dieta artificial à base de feijão, germe de trigo e farelo de soja de acordo comGreENE etal.(1976). Após o resfriamento da dieta, as lagartas foram transferidas para o tubo, sendo mantidas nestes recipientes até o período pupal.

Obtenção e multiplicação dos isolados deBacillus thuringiensis (B.t). Foram utilizados 27 isolados de $B$. thuringiensis oriundos de amostras de solo e dois formulados comerciais à base de $B$. thuringiensis var. aizawai (Xentari ${ }^{\circledR}$ WG) e à base de $B$. thuringiensis var. kurstaki (Dipel $\left.{ }^{\circledR} \mathrm{PM}\right)$. Os isolados utilizados neste experimento foram provenientes da ESALQ/USP, os quais fazem parte do banco de entomopatógenos do NUDEMAFI do CCA-UFES. A multiplicação dos isolados foi realizada em meio LB - Luria-Bertani (extrato de levedura, peptona, cloreto de sódio, ágar e água destilada), procedendo com o isolamento da bactéria (papel filtro), por um período de 24 a 48 horas e, depois, sua multiplicação em placas cheias, durante 72 horas; ambas as fases foram realizadas em temperatura de $28^{\circ} \mathrm{C}$. Posteriormente, as colônias foram removidas das placas com auxílio de espátula e transferidas para tubos plásticos, contendo $10 \mathrm{~mL}$ de água destilada estéril. Com o objetivo de eliminar as toxinas indesejáveis e restos de meio de cultura, procedeu-se a centrifugação decada isolado por $20 \mathrm{minu}$ tos por três vezes (5.000 rpm) consecutivas, assim como do material proveniente do produto Xentari ${ }^{\circledR}$ WG (Bta). Após a última centrifugação, o material de cada isolado foi ressuspenso em água destilada estéril e a concentração foi ajustada para $3 \times 10^{8}$ esporos / $\mathrm{mL}^{-1}$ com auxílio de câmara de Neubauer ${ }^{\circledR}$ e microscópio óptico. A concentração de Dipel ${ }^{\circledR} \mathrm{PM}$ (Btk) foi ajustada para $3 \times 10^{8}$ esporos $\mathrm{mL}^{-1}$ de acordo especificação do fabricante, procedimento que não foi possível ser adotado para o produto Xentari ${ }^{\circledR}$ WG, por não possuir a especificação de ingrediente ativo/ grama, o que tornou necessário isolar a bactéria e adotar a metodologia empregada para a multiplicação dos isolados, utilizando o meio LB - Luria-Bertani (extrato de levedura, peptona, cloreto de sódio, ágar e água destilada), como descrito anteriormente no processo de multiplicação dos isolados.

Suscetibilidade de Helicoverpa zea ao Bacillus thuringiensis. Os ensaios foram realizados com bandejas confeccionadas com microtubos de acrílico de $3,0 \mathrm{~cm} \times 2,0 \mathrm{~cm}$ de diâmetro, onde $1 / 4$ dos tubos foram preenchidos com a mesma dieta artificial, utilizada na fase de criação de $H$. zea. Posteriormente, $50 \mu \mathrm{L}$ de cada isolado na concentração $3 \times 10^{8}$ esporos $\mathrm{mL}^{-1}$ foi inoculado nos microtubos de acrílico contendo dieta artificial. Após a evaporação do excesso de umidade, 50 lagartas de primeiro instar foram acondicionadas individualmente, perfazendo desta forma 50 repetições por tratamento. $\mathrm{O}$ mesmo procedimento foi realizado para a testemunha, mas utilizando somente água destilada estéril sobre a dieta.

O experimento foi realizado em delineamento inteiramente casualizado com 30 tratamentos e 50 repe- 
tições, perfazendo1.500 insetos, sendo conduzido em sala climatizada $\left(26 \pm 1^{\circ} \mathrm{C}, 70 \pm 10 \%\right.$ de UR e fotofase de $12 \mathrm{~h}$ ) e avaliado até o $7^{\circ}$ dia. A patogenicidade dos isolados e dos produtos à base de B.t sobre as lagartas foi avaliada diariamente, determinando a mortalidade e o tempo letal $\left(\mathrm{TL}_{50}\right)$, os quais foram aferidos em intervalos de 12, 24, 48,72 e 96h; para a análise foram considerados apenas os isolados que apresentaram mortalidade superior a $90 \%$. Os dados foram submetidos à análise de variância e as médias comparadas pelo teste de Scoot-Knot a 5\% de probabilidade, utilizando o programa SAEG (versão 5.0). O $\mathrm{TL}_{50}$ foi determinado através do programa Micro-probit (versão 3.0) (HADDAD et al., 1995).

Efeito subletal de Bacillus thuringiensis a Helicoverpa zea. Com o objetivo de observar possíveis efeitos subletais nos insetos sobreviventes, foi realizada a pesagem das lagartas e, posteriormente, das pupas. A pesagem das lagartas e de pupas foi realizada em balança analítica devidamente calibrada, onde um grupo de 20 insetos de cada tratamento que apresentou mortalidade inferior a $34 \%$ foi escolhido aleatoriamente. Os insetos foram individualizados em tubos de vidro similares aos utilizados para criação da praga e, ao atingirem o estágio pupal, foram novamente pesados. Os dados foram submetidos à análise de variância e as médias comparadas pelo teste de Scoot-Knot $(\mathrm{P}=0,05)$ a 5\% de probabilidade, utilizando o programa de análise estatística SAEG (versão 5.0).

Estimativa da Concentração Letal $\left(\mathrm{CL}_{50}\right)$. Os isolados de B.t que proporcionaram mortalidade superi- or a $90 \%$, assim como o produto Dipel ${ }^{\circledR} \mathrm{PM}$, foram submetidos a bioensaios para estimativa da $\mathrm{CL}_{50}$ onde foram utilizadas 100 lagartas de primeiro instar por tratamento, e as condições experimentais foram as mesmas do teste de suscetibilidade. Para cada isolado foram testadas seis concentrações, as quais foram estabelecidas em ensaios preliminares; para a testemunha utilizou-se água destilada estéril sobre a dieta. Cada tratamento foi composto por 700 lagartas, perfazendo 5.600 insetos. Os bioensaios foram avaliados diariamente, até o $5^{\circ}$ dia após a aplicação. As $\mathrm{CLs}_{50}$ foram estimadas com auxílio do programa computacional Mobae, através do Micro-probit (versão 3.0) (HADDAD et al., 1995).

\section{RESULTADOS E DISCUSSÃO}

Suscetibilidade de $H$. zea a $B$. thuringiensis. A patogenicidade dos isolados de $B$. thuringiensis e os produtos Xentari ${ }^{\circledR}$ WG e Dipel ${ }^{\circledR}$ PM apresentaram resultados significativos $(\mathrm{P}=0,05 ; \mathrm{F}=163,78 ; \mathrm{Gl}=28)$, sendo que apenas os isolados B.t 11, B.t 21, B.t 23, B.t 25, B.t 26, B.t 27, B.t 28 e os produtos comerciais proporcionaram mortalidades superiores a $94,1 \%$; os demais isolados não apresentaram percentuais satisfatórios no controle de $H$. zea na concentração utilizada, com mortalidades inferiores a 33,3\% (Tabela 1). Estes resultados denotam a importância da realização de testes de patogenicidade, pois permitem a identificação de isolados eficientes contra o inseto-alvo.

Tabela 1 - Mortalidade de H. zea por diferentes isolados de B. thuringiensis e dois formulados comerciais Xentari ${ }^{\circledR}$ WG e Dipel ${ }^{\circledR}$ PM. Temperatura: $26 \pm 1^{\circ} \mathrm{C}$, UR de $65 \pm 5 \%$ e fotofase de $12 \mathrm{~h}$.

\begin{tabular}{lccc}
\hline Tratamentos & Mortalidade & Tratamentos & Mortalidade \\
\hline B.t 21 & $100 \pm 0,00 \mathrm{~A}$ & B.t 10 & $9,8 \pm 0,04 \mathrm{C}$ \\
B.t 23 & $100 \pm 0,00 \mathrm{~A}$ & B.t 13 & $9,8 \pm 0,04 \mathrm{C}$ \\
B.t 25 & $100 \pm 0,00 \mathrm{~A}$ & B.t 14 & $9,8 \pm 0,04 \mathrm{C}$ \\
B.t 26 & $100 \pm 0,00 \mathrm{~A}$ & B.t 18 & $7,8 \pm 0,04 \mathrm{C}$ \\
B.t 27 & $100 \pm 0,00 \mathrm{~A}$ & B.t 17 & $5,9 \pm 0,03 \mathrm{D}$ \\
B.t 28 & $98,0 \pm 0,02 \mathrm{~A}$ & B.t 1 & $3,9 \pm 0,03 \mathrm{D}$ \\
Bta & $98,0 \pm 0,02 \mathrm{~A}$ & B.t 2 & $3,9 \pm 0,03 \mathrm{D}$ \\
Btk & $98,0 \pm 0,02 \mathrm{~A}$ & B.t 5 & $3,9 \pm 0,03 \mathrm{D}$ \\
B.t 11 & $94,1 \pm 0,03 \mathrm{~A}$ & B.t 6 & $3,9 \pm 0,03 \mathrm{D}$ \\
B.t 24 & $33,3 \pm 0,07 \mathrm{~B}$ & B.t 7 & $3,9 \pm 0,03 \mathrm{D}$ \\
B.t 16 & $15,7 \pm 0,05 \mathrm{C}$ & B.t 9 & $3,9 \pm 0,03 \mathrm{D}$ \\
B.t 20 & $13,7 \pm 0,05 \mathrm{C}$ & B.t 3 & $1,9 \pm 0,02 \mathrm{D}$ \\
B.t 15 & $11,7 \pm 0,05 \mathrm{C}$ & B.t 4 & $1,9 \pm 0,02 \mathrm{D}$ \\
B.t 19 & $11,7 \pm 0,05 \mathrm{C}$ & B.t 12 & $0,0 \pm 0,00 \mathrm{D}$ \\
B.t 8 & $9,8 \pm 0,04 \mathrm{C}$ & Testemunha & $0,0 \pm 0,00 \mathrm{D}$ \\
\hline
\end{tabular}

${ }^{1}$ Médias ( \pm EP) seguidas de mesma letra maiúsculas na coluna não diferem entre si, pelo teste de Scoot-Knot, ao nível de $5 \%$ de significância. 
Tabela 2 - Tempo letal $\left(\mathrm{TL}_{50}\right)$ em horas e intervalos de confiança para lagartas de $1^{\circ}$ instar de $H$. zea infectadas por $B$. thuringiensis. Temperatura: $26 \pm 1^{\circ} \mathrm{C}$, UR de $65 \pm 5 \%$ e fotofase de $12 \mathrm{~h}$.

\begin{tabular}{llll}
\hline Tratamentos & \multicolumn{1}{c}{ Equação de regressão } & $\mathrm{TL}_{50}\left(\mathrm{IC}_{0,05}\right)$ & $\chi^{2(1)}$ \\
\hline B.t 11 & $\mathrm{y}=(4,0656)+1,3409 * \log \mathrm{x}$ & $4,97(0,93-9,44)$ & 0,43 \\
B.t 21 & $\mathrm{y}=(2,9433)+2,0609 * \log \mathrm{x}$ & $9,95(5,69-13,64)$ & 0,94 \\
B.t 23 & $\mathrm{y}=(3,6710)+1,9393 * \log \mathrm{x}$ & $4,84(0,95-8,51)$ & 0,92 \\
B.t 25 & $\mathrm{y}=(2,8809)+2,5303 * \log \mathrm{x}$ & $6,87(2,02-10,15)$ & 0,06 \\
B.t 26 & $\mathrm{y}=(3,5935)+2,0409 * \log \mathrm{x}$ & $4,88(0,77-8,58)$ & 1,49 \\
B.t 27 & $\mathrm{y}=(2,4594)+2,8209 * \log \mathrm{x}$ & $7,95(3,26-10,94)$ & 0,16 \\
B.t 28 & $\mathrm{y}=(3,6496)+1,8224 * \log \mathrm{x}$ & $5,50(1,63-9,20)$ & 0,20 \\
Btk & $\mathrm{y}=(3,5845)+1,7669 * \log \mathrm{x}$ & $6,32(2,44-10,08)$ & 0,85 \\
Bta & $\mathrm{y}=(3,7795)+1,5083 * \log \mathrm{x}$ & $6,44(2,33-10,63)$ & 2,38 \\
\hline
\end{tabular}

$\mathrm{n}=50$ insetos/tratamento.

${ }^{1}$ Qui-quadrado não significativo.

Tabela 3 - Efeito subletal de diferentes isolados de B. thuringiensis no peso de lagartas e pupas de H. zea. Temperatura: $26 \pm 1^{\circ} \mathrm{C}$, UR de $65 \pm 5 \%$ e fotofase de $12 \mathrm{~h}$.

\begin{tabular}{|c|c|c|c|}
\hline Tratamentos & Peso (g) & Tratamentos & Peso $(\mathrm{g})$ \\
\hline & Lagartas & \multicolumn{2}{|c|}{ Pupas } \\
\hline Testemunha & $0,0255 \pm 0,0011 \mathrm{~A}$ & Testemunha & $0,5212 \pm 0,01 \mathrm{~A}$ \\
\hline B.t 1 & $0,0258 \pm 0,0031 \mathrm{~A}$ & B.t 1 & $0,5151 \pm 0,01 \mathrm{~A}$ \\
\hline B.t 2 & $0,0238 \pm 0,0035 \mathrm{~A}$ & B.t 2 & $0,5062 \pm 0,01 \mathrm{~A}$ \\
\hline B.t 3 & $0,0262 \pm 0,0021 \mathrm{~A}$ & B.t 3 & $0,5241 \pm 0,01 \mathrm{~A}$ \\
\hline B.t 7 & $0,0263 \pm 0,0014 \mathrm{~A}$ & B.t 4 & $0,5186 \pm 0,02 \mathrm{~A}$ \\
\hline B.t 8 & $0,0255 \pm 0,0020 \mathrm{~A}$ & B.t 5 & $0,5127 \pm 0,01 \mathrm{~A}$ \\
\hline B.t 9 & $0,0263 \pm 0,0018 \mathrm{~A}$ & B.t 7 & $0,5201 \pm 0,01 \mathrm{~A}$ \\
\hline B.t 10 & $0,0243 \pm 0,0018 \mathrm{~A}$ & B.t 8 & $0,5186 \pm 0,01 \mathrm{~A}$ \\
\hline B.t 13 & $0,0262 \pm 0,0018 \mathrm{~A}$ & B.t 10 & $0,5066 \pm 0,01 \mathrm{~A}$ \\
\hline B.t 14 & $0,0259 \pm 0,0015 \mathrm{~A}$ & B.t 17 & $0,5037 \pm 0,02 \mathrm{~A}$ \\
\hline B.t 19 & $0,0261 \pm 0,0019 \mathrm{~A}$ & B.t 20 & $0,5024 \pm 0,02 \mathrm{~A}$ \\
\hline B.t 4 & $0,0190 \pm 0,0025 \mathrm{~B}$ & B.t 24 & $0,5265 \pm 0,01 \mathrm{~A}$ \\
\hline B.t 5 & $0,0204 \pm 0,0022 \mathrm{~B}$ & B.t 6 & $0,4744 \pm 0,04 \mathrm{~B}$ \\
\hline B.t 12 & $0,0221 \pm 0,0019 \mathrm{~B}$ & B.t 9 & $0,4783 \pm 0,02 \mathrm{~B}$ \\
\hline B.t 17 & $0,0206 \pm 0,0023 \mathrm{~B}$ & B.t 12 & $0,4758 \pm 0,01 \mathrm{~B}$ \\
\hline B.t 15 & $0,0136 \pm 0,0011 \mathrm{C}$ & B.t 13 & $0,4552 \pm 0,01 B$ \\
\hline B.t 16 & $0,0122 \pm 0,0010 \mathrm{C}$ & B.t 14 & $0,4893 \pm 0,01 \mathrm{~B}$ \\
\hline B.t 18 & $0,0165 \pm 0,0023 \mathrm{C}$ & B.t 15 & $0,4623 \pm 0,02 \mathrm{~B}$ \\
\hline B.t 20 & $0,0110 \pm 0,0012 \mathrm{C}$ & B.t 16 & $0,4742 \pm 0,02 \mathrm{~B}$ \\
\hline B.t 6 & $0,0036 \pm 0,0002 \mathrm{D}$ & B.t 18 & $0,4813 \pm 0,01 \mathrm{~B}$ \\
\hline B.t 24 & $0,0051 \pm 0,0005 \mathrm{D}$ & B.t 19 & $0,4576 \pm 0,02 \mathrm{~B}$ \\
\hline
\end{tabular}

${ }^{1}$ Médias ( \pm EP) seguidas de mesma letra maiúscula na coluna não diferem entre si, pelo teste de Scoot-Knot, ao nível de $5 \%$ de significância.

O isolado B.t12nãoapresentou nenhuma virulência para H. zea (Tabela 1), isso pode ser relacionado à falta de toxicidade do isolado. Estudos de Rogoff etal. (1969) verificaram baixa mortalidade de H. zea ao avaliar isolados de B.t, dentre estes EPE-2000 de B. thuringiensis var. thuringiensis e o isolado HA-3 de B. thuringiensis var. aizawai. AguILAR-Medel et al. (2007) verificaram variabilidade de quatro populações distintas de $H$. zea em relação a sua suscetibilidade à $\delta$-endotoxina de $B$. thuringiensisem diferentes concentrações, sendo que na concentração mais alta a mortalidade foi inferior a $43 \%$. 
Tabela 4 - Estimativa da $\mathrm{CL}_{50}$ de diferentes isolados e um formulado de $B$. thuringiensisem lagartas de H. zea. Temperatura: $26 \pm 1^{\circ} \mathrm{C}$, UR de $65 \pm 5 \%$ e fotofase de $14 \mathrm{~h}$.

\begin{tabular}{llll}
\hline Tratamentos & \multicolumn{1}{c}{ Equação } & \multicolumn{1}{c}{$\mathrm{CL}_{50}\left(\mathrm{IC}_{0,05}\right)$} & $\chi^{\left({ }^{*}\right)}$ \\
\hline B.t 11 & $\mathrm{y}=(-8,4609)+1,9906^{*} \log \mathrm{x}$ & $5,7 \times 10^{6} ;\left(6,6 \times 10^{6} ; 5 \times 10^{6}\right)$ & 1,05 \\
B.t 21 & $\mathrm{y}=(-6,9578)+1,9087^{*} \log \mathrm{x}$ & $1,8 \times 10^{6} ;\left(2,1 \times 10^{6} ; 1,5 \times 10^{6}\right)$ & 4,30 \\
B.t 23 & $\mathrm{y}=(0,6654)+0,9679 * \log \mathrm{x}$ & $3 \times 10^{4} ;\left(4 \times 10^{4} ; 2,2 \times 10^{4}\right)$ & 6,63 \\
B.t 25 & $\mathrm{y}=(-1,4345)+1,2293^{*} \log \mathrm{x}$ & $1,7 \times 10^{5} ;\left(2,2 \times 10^{5} ; 1,3 \times 10^{5}\right)$ & 1,13 \\
B.t 26 & $\mathrm{y}=(-1,9206)+1,2715^{*} \log \mathrm{x}$ & $2,7 \times 10^{5} ;\left(3,7 \times 10^{5} ; 2 \times 10^{5}\right)$ & 3,19 \\
B.t 27 & $\mathrm{y}=(-1,7649)+1,2278^{*} \log \mathrm{x}$ & $3,2 \times 10^{5} ;\left(4,4 \times 10^{5} ; 2,4 \times 10^{5}\right)$ & 6,05 \\
B.t 28 & $\mathrm{y}=(-0,2815)+0,8665^{*} \log \mathrm{x}$ & $1,2 \times 10^{6} ;\left(1,8 \times 10^{6} ; 7,8 \times 10^{5}\right)$ & 6,91 \\
Btk & $\mathrm{y}=(-3,7712)+1,2374 * \log \mathrm{x}$ & $1,2 \times 10^{7} ;\left(1,5 \times 10^{7} ; 9,7 \times 10^{6}\right)$ & 3,83 \\
\hline
\end{tabular}

$\mathrm{N}=100$ insetos/tratamento.

${ }^{*}$ n.s = não significativo.

Os valores obtidos para $\mathrm{TL}_{50}$ variaram entre $4,84 \mathrm{a}$ $9,95 \mathrm{~h}$ entre os isolados que apresentaram mortalidade superior a $90 \%$ (Tabela 2), sendo que para Dipel ${ }^{\circledR}$ PM e Xentari ${ }^{\circledR}$ WG os valores foram de 6,32 e 6,44h, respectivamente. A importância dessa análise pode ser correlacionada com a eficiência do isolado, pois aqueles que apresentam valores menores de TL conseguem matar o inseto-alvo mais rapidamente, sendo um dos pontos importantes a serem considerados na seleção. De acordo com a TL ${ }_{50}$, os isolados B.t 23 e B.t 26 foram os mais eficientes (Tabela 2).

Efeito Subletal de $B$. thuringiensis a $H$. zea. Os isolados B.t 4, B.t 5, B.t 6, B.t 12, B.t 15, B.t16, B.t17, B.t 18 , B.t 20 e B.t 24 promoveram a redução no peso das lagartas sendo significativamente inferior a testemunha $(\mathrm{P}=0,05 ; \mathrm{F}=13,69 ; \mathrm{Gl}=20)$ (Tabela 3). Resultados similares foram encontrados por EIZAGUIRRE et al.(2005), ao avaliarem o efeito subletal de $B$. thuringiensis sobre o desenvolvimento larval de Sesamia nonagrioides (Lefèvbre) (Lepidoptera: Noctuidae) e por PoLANCZYK; Alves (2005) ao verificarem o efeito subletal dealguns isolados de B. thuringiensis a lagartas de Spodoptera frugiperda (J.E. Smith) (Lepidoptera: Noctuidae).

MASCARENHAS; LutTRELl (1997) observaram redução no peso de lagartas de $H$. zea alimentadas com algodão B.t, o qual expressa a endotoxina Cry1A de B. thuringiensis, em comparação aos insetos alimentados com uma cultivar convencional de algodão.

Pedersen et al. (1997) e Moreau; Bauce (2003) verificaram que doses subletais de $B$. thuringiensis afetaram o desenvolvimento de Choristoneura fumiferana (Clemens) (Lepidoptera: Tortricidae), reduzindo o peso das pupas. Assim como os trabalhos citados anteriormente, evidenciou-se a redução no peso das pupas $(\mathrm{P}=0,05 ; \mathrm{F}=1,46 ; \mathrm{Gl}=20)$ de H. zeatratadascom os isolados B.t 6, B.t 9, B.t12, B.t13, B.t14, B.t15, B.t16, B.t 18 e B.t 19. Sendo que B.t 1, B.t 2, B.t3, B.t 4, B.t 7, B.t 8 e B.t 10 não afetaram o desenvolvimento larval e pupal de H. zea (Tabela3). Resultados similares foram encontrados por ERB et al. (2001) ao avaliarem o efeito subletal de $B$. thuringiensis sobre o desenvolvimento de Lymantria dispar (Linnaeus) (Lepidoptera: Lymantriidae).

Estimativa da Concentração Letal Média $\left(\mathrm{CL}_{50}\right)$. A concentração requerida para ocasionar a mortalidade de $50 \%$ da população de $H$. zea variou entre $3 \times$ $10^{4}$ a $1,2 \times 10^{7}$ esporos $\mathrm{mL}^{1}$ de $B$. thuringiensis, sendo que o isolado B.t 23 foi o mais virulento, apresentando intervalo de confiança de $4 \times 10^{4}(\mathrm{P}=0,05)$ e $2,2 \times 10^{4}$ esporos $\mathrm{mL}^{1}(\mathrm{P}=0,05)$ de B.t (Tabela 4$)$. De acordo o intervalo de confiança houve resposta semelhante entre os isolados B.t 26 e B.t 27 (Tabela 4), apresentando toxicidade semelhante para as lagartas deH. zea de acordo os limites superiores e inferiores da $\mathrm{CL}_{50}$ (Tabela 3). Em relação ao produto Dipe ${ }^{\circledR} \mathrm{PM}$ foi necessária concentração de 1,2 x $10^{7}$ esporos $\mathrm{mL}^{1}$ para ocasionar morte em $50 \%$ da população de H. zea (Tabela 4).

AMEen et al. (1998) avaliaram a patogenicidade dos formulados Dipel ES ${ }^{\circledR}$, Dipel 6AF ${ }^{\circledR}$ e Xentari AS ${ }^{\circledR}$ a lagartas de $H$. zea, sendo queXentari $A S^{\circledR}$ apresentou a $\mathrm{CL}_{50}$ superior aos demais, similarmente ao resultado encontrado neste trabalho (Tabela 4). AguILAR-MENDEL et al. (2007) avaliaram a suscetibilidade de diferentes populações de $H$. zea a $\delta$-endotoxina Cry2Ab através da análise de probit, concluindo que houve variação entre as populações avaliadas e $\mathrm{CL}_{50}$ variaram entre 0,017 a $0,086 \mu \mathrm{g}$ da $\delta$-endotoxina $\mathrm{mL}^{1}$ de dieta.

O isolado B.t 23 pode ser considerado promissor para o controle de lagartas de $H$. zea, uma vez que apresentou maior virulência em comparação aos demais. No entanto, estudos mais detalhados são necessários para validar a sua utilização no manejo de $H$. zea, tais como: caracterização, produção em larga escala; virulência a outros insetos-praga e efeitos sobre organismos benéficos, uma vez que o comportamento desse isolado em condições de campo pode sofrer variações mediante a influência de fatores bióticos e abióticos. 


\section{CONCLUSÕES}

Os isolados B.t 1 a B.t 10, B.t 12 a B.t 20 apesar de pouco virulentos afetam o desenvolvimento deH.zea.

Os isolados B.t 11, B.t 21, B.t 23, B.t 25, B.t 26, B.t 27 e B.t 28 foram mais virulentos a $H$. zea; Mediante os resultados da CL50 e TL50 o isolado Bt 23 é considerado promissor no controle de $H$. zea.

\section{AGRADECIMENTOS}

ÀUFRPEe ao CCA-UFES, por permitirem o desenvolvimento dessa pesquisa. À CAPES, por ter concedido a bolsa de estudos ao primeiro autor.

\section{REFERÊNCIAS}

AGUILAR-MEDEL, S.; RODRÍGUEZ-MACIEL, J.C.; DÍAZ-GÓMEZ, O.; MARTÍNEZ-CARRILLO, J.L.; LÓPEZ-COLLADO, J.; BLANCO, C.A.; LAGUNESTEJEDA, A. Susceptibilidad de Helicoverpa zea (Boddie) a la $\delta$-endotoxina $\mathrm{Cry} 2 \mathrm{Ab}$ de Bacillus thuringiensis Berliner. Agrociencia, v.41, n.6, p.653-662, 2007.

AMEEN, A.O.; FUXA, J.R.; RITCHER, A.R. Antagonism between formulations of different Bacillus thuringiensis subspecies in Heliothis virescens and Helicoverpa zea (Lepidoptera: Noctuidae). Journal of Entomological Science, v.33, n.2, p.129-135, 1998.

BOBROWSKI, V.L.; FIÚZA, L.M.; PASQUALI, G.; BODANESE-ZANETTINI, M.H. Genes de Bacillus thuringiensis: uma estratégia para conferir resistência a insetos em plantas. Ciência Rural, v.34, n.1, p.843-850, 2003.

EIZAGUIRRE, M.; TORT, S.; LÓPEZ, C.; ALBAJES, R. Effects of sublethal concentrations of Bacillus thuringiensis on larval development of Sesamia nonagrioides. Journal of Economic Entomology, v.98, n.2, p.464-470, 2005.

ERB, S.L.; BOURCHIER, R.S.; van FRANKENNHUYZEN, K.; SMITH, S.M. Sublethal effects of Bacillus thuringiensis subsp. kurstaki on Lymantria dispar (Lepidoptera: Lymantriidae) and the tachinid parasitoid Compsilura concinnata (Diptera: Tachinidae). Environmental Entomology, v.6, n.30, p.11741181, 2001.

ESCUDERO, I.R.; IBÁÑEZ, I.; PADILLA, M.A.; CARNERO, A.; CABALLERO, P. Aislamiento y caracterización de nuevas cepas de Bacillus thuringiensis procedentes de muestras de tierra de Canarias. Boletin de Sanidad Vegetal. Plagas, v.30, n.4, p.703-712, 2004.

FRANÇA, F. H.; VILLASBÔAS, G. L.; CASTELOBRANCO, M.; MEDEIROS, M. A. Manejo integrado de pragas. In: SILVA, J.B.C.; GIORDANO, L.B. (Org.). Tomate para processamento industrial. Brasília: Embrapa-CNPH/Embrapa Comunicação para Transferência de Tecnologia, 2000. p.112-127.

GIOLO, F.P.; BUSATO, G.R.; GARCIA, M.S.; MANZONI, C.G.; BERNADI, O.; ZART, M. Biologia de Helicoverpa zea (Boddie, 1850) (Lepidoptera: Noctuidae) em duas dietas artificiais. Revista Brasileira de Agrociência, v.12, n.2, p.167-171, 2006.

GLARE, T.R.; O'CALLAGHAN, M. Bacillus thuringiensis: biology, ecology and safety. Chichester: John Wiley \& Sons, 2000. 350p.

GOULD, F.; BLAIR, N.; REID, M.; RENNIE, T.L.; LOPEZ, J.; MICINSKI, S. Bacillus thuringiensis-toxin resistance management: Stable isotope assessment of alternate host use by Helicoverpa zea. Proceedings of the National Academy of Sciences of the United States of America, v.99, n.26, p.16581-16586, 2002.

GREENE, G.L.; LEPPLA, N.C.; DICKERSON, W.A. Velvetbean caterpillar: a rearing procedure and artificial médium. Journal Economic Entomology, v.69, n.4, p.487-488, 1976.

HADDAD, M.L.; MORAES, R.C.B.; PARRA, J.R.P. Programa MOBAE: Modelos bioestatísticos aplicados à entomologia.(software). Piracicaba: ESALQ/USP, 1995. $44 \mathrm{p}$.

KARIM, S.; RIAZUDDIN, S., GOULD, F.; DEAN, D. H. Determination of receptor binding properties of Bacillus thuringiensis ä-endotoxins to cotton bollworm (Helicoverpa zea) and pink bollworm (Pectinophora gossypiella) midgut brush border membrane vesicles. Pesticide Biochemistry and Physiology, v.67, n.3, p.198-216, 2000.

LEBEDENCO, A.; AUAD, A.M.; KRONKA, S.N. Métodos de controle de lepidópteros do tomateiro (Lycopersicon esculentum Mill.). Acta Scientiarum Agronomy, v.29, n.3, p.339-344, 2007.

MASCARENHAS, V.J.; LUTTRELL, R.G. Combined effect of sublethal exposure to cotton expressing the endotoxin protein of Bacillus thuringiensis and natural enemies on survival of Bollworm (Lepidoptera:

Noctuidae) larvae. Environmental Entomology, v.26, n.4, p.939-945, 1997.

MEDEIROS, P.T; FERREIRA, M.C.; MARTINS, E.S.; GOMES, A.C.M.M.; FALCÃO, R.; SOUZA DIAS, J.M.C.; MONNERAT, R.G. Seleção e caracterização de estirpes de Bacillus thuringiensis efetivas no controle da traçadas-crucíferas Plutella xylostella. Pesquisa Agropecuária Brasileira, v.40, n.11, p.1145-1148, 2005.

MOREAU, G.; BAUCE, E. Lethal and sublethal effects of single and double applications of Bacillus thuringiensis 
variety kurstaki on spruce budworm (Lepidoptera: Tortricidae) larvae. Journal Economic Entomology, v.96, n.2, p.280-286, 2003.

NAULT, B.A.; SPEESE III, J. Major insects pests and economics of fresh-market tomato in eastern Virginia. Crop Protection, v.21, n.5, p.359-366, 2002.

PINTO, A. S.; PARRA, J. R. P.; OLIVEIRA, H.N. Pragas e insetos benéficos do milho. Piracicaba: Esalq/USP, 2004. $108 \mathrm{p}$.

PEDERSEN, A.; DEDES, J.; D. GAUTHIER D.; VAN FRANKENHUYZEN K. Sublethal effects of Bacillus thuringiensis on the spruce budworm, Choristoneura fumiferana. Entomologia Experimentalis et Applicata, v.83, n.3, p.253-262, 1997.

POLANCZYK, R.A.; ALVES, S.B. Bacillus thuringiensis: Uma breve revisão. Agrociencia, v.7, n.2, p.1-10, 2003.
POLANCZYK, R.A.; ALVES, S.B. Biological parameters of Spodoptera frugiperda (J.E. Smith) (Lepidoptera: Noctuidae) assayed with Bacillus thuringiensis Berliner. Scientia Agrícola, v.62, n.5, p.464-468, 2005.

POLANCZYK, R.A.; VALICENTE, F.H.; BARRETO, M.R. Utilização de Bacillus thuringiensis no controle de pragas agrícolas na América Latina. In ALVES, S.B.; LOPES, R.B. (Ed.). Controle microbiano de pragas na América Latina: avanços e desafios. Piracicaba, FEALQ, 2008. Cap. 4, 414p.

ROGOFF, M.H.; IGNOFFO, C.M.; SINGER, S.; I. GARD; PRIETO, A.P. Insecticidal activity of thirty-one strains of Bacillus thuringiensis five insect species. Journal of Invertebrate Pathology, v.14, n.2, p.122-129, 1969.

Recebido em 13/2/09

Aceito em 5/10/09 